\title{
El papel de los biosimilares en la enfermedad inflamatoria intestinal: una realidad en nuestro país
}

\author{
The Role of Biosimilars in Inflammatory Bowel Disease: A Reality in our \\ Country
}

Juan Ricardo Márquez, ${ }^{1}$ Fernando Gomollón. ${ }^{2}$

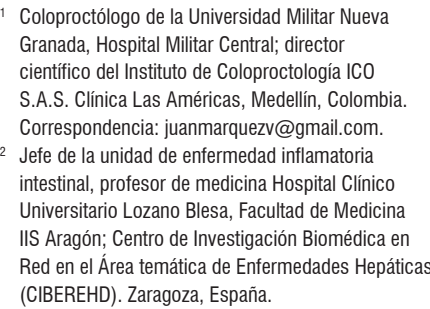

Coloproctólogo de la Universidad Militar Nueva Granada, Hospital Militar Central; director científico del Instituto de Coloproctología ICO S.A.S. Clínica Las Américas, Medellín, Colombia. Correspondencia: juanmarquezv@gmail.com.

2 Jefe de la unidad de enfermedad inflamatoria intestinal, profesor de medicina Hospital Clínico Universitario Lozano Blesa, Facultad de Medicina IIS Aragón; Centro de Investigación Biomédica en Red en el Área temática de Enfermedades Hepáticas (CIBEREHD). Zaragoza, España.

Fecha recibido: $\quad 15-03-17$

Fecha aceptado: 06-10-17

\begin{abstract}
Resumen
La enfermedad inflamatoria intestinal (EII) es un término con el que se conocen varias entidades, las 2 más importantes son: la colitis ulcerativa idiopática (CUI) y la enfermedad de Crohn (EC), cuyo origen es multifactorial y se caracterizan por un fenómeno inflamatorio, crónico, recurrente con diferentes grados de severidad del tubo digestivo; pero además con afectación potencial de otros órganos.

En la última década ha habido un renovado interés en dichas entidades, debido a una incidencia creciente de estas, pero también debido al desarrollo de medicamentos que por primera vez están cambiando la historia natural de estas enfermedades: son los medicamentos llamados biológicos, que son aquellos producidos o derivados de organismos vivos y representan el sector de mayor crecimiento en la industria farmacéutica mundial. Debido al interés comercial, se han desarrollado y sometido a las autoridades reguladoras productos no originadores; similares, pero no idénticos a los productos de referencia.

La Agencia Europea de Medicamentos (EMA) aprobó el primer biosimilar del infliximab para todas las indicaciones en las cuales este estaba aprobado, incluidas: colitis ulcerativa moderada a severa en todas las edades, EC moderada a severa en todas las edades, al igual que EC fistulizante, artritis psoriásica (AP), psoriasis, espondilitis anquilosante (EA) y artritis reumatoide (AR) (1).

En esta revisión nos proponemos definir lo que son estos productos al igual que algunos conceptos relacionados (extrapolación, sustitución e intercambiabilidad), además hacer una revisión histórica de su desarrollo, sus indicaciones actuales, las posiciones de las diferentes asociaciones científicas al respecto y, lo más importante, brindar datos de la vida real en cuanto a su efectividad, seguridad y costos en los diferentes países donde se han estado usando.
\end{abstract}

\section{Palabras clave}

Biosimilares; agentes biológicos; anti-TNF; extrapolación, sustitución e intercambiabilidad.
Los medicamentos biológicos (aquellos producidos o derivados de organismos vivos) representan el sector de mayor crecimiento en la industria farmacéutica mundial. En el 2007, la industria farmacéutica global de los biológicos rondaba los 100000 millones de dólares, pero en tan solo 10 años ha crecido tanto que para el 2017 las ventas de estos productos se estiman en el $20 \%$ del total del mercado farmacéutico mundial y para el 2020 ascenderá a 250000 millones de dólares (2). En el 2008, globalmente 2 de los 10 medicamentos (top ten) más vendidos anualmente eran biológicos, pero para el 2016 esta cifra ascendió a 7; en este mismo período, el número de biológicos aprobados por la Administración Federal de Drogas y Alimentos (FDA) ascendió anualmente del 33\% al 60\%, siendo Estados Unidos el país que más los consume con el $50 \%$ de las ventas mundiales (3).

En Colombia en 2012, 8 de los diez medicamentos no incluidos en el plan de beneficios con mayores recobros al Estado eran de origen biológico y en 2013, aproximada- 


\begin{abstract}
The term Inflammatory Bowel Disease (IBD) is used to identify several entities, the two most important of which are Idiopathic Ulcerative Colitis (IUC) and Crohn's Disease (EC). Both are multifactorial in origin, are chronic and recurrent, are characterized by inflammation, have varying degrees of severity, and potentially involve other organs.

In the last decade there has been renewed interest in these entities due to growing incidence and to the development of drugs called biologicals. For the first time, these drugs have changed the natural history of these diseases. They are produced or derived from living organisms and represent the fastest growing sector in the global pharmaceutical industry. Due to commercial interest, products similar but not identical to the original products have been developed and submitted to regulatory authorities.

The European Medicines Agency has approved the first biosimilar for infliximab for all indications for which it has been approved. These include moderate to severe ulcerative colitis (UC) at all ages, moderate to severe Crohn's disease (CD) at all ages, fistulizing Crohn's disease, Psoriasic arthritis, Psoriasis, Ankylosing Spondylitis, and Rheumatoid Arthritis.

In this review, we define what these products are, as well as some concepts coined for their usage including extrapolation, substitution and interchangeability. We also make a historical review of their development, current indications, the positions of various scientific associations with respect to them, and - most importantly - provide real-life data regarding their effectiveness, safety and costs in countries where they have been used.
\end{abstract}

\title{
Key words
}

Biosimilar; biological agents; anti TNF; extrapolation; substitution and interchangeability.

mente el 36\% (USD \$1250 millones) del mercado total farmacéutico nacional correspondió a biológicos (4).

Debido al interés comercial, se han desarrollado y sometido a las autoridades reguladoras productos no originadores; similares, pero no idénticos a los productos de referencia debido a que estos son estructuras grandes, complejas y la variabilidad inherente no solo al proceso de manufactura sino también a la naturaleza misma del biológico hacen que sea imposible crear una molécula idéntica. Entre otras, las razones son: vencimiento de las patentes de los primeros biológicos pioneros, la crisis financiera mundial (reducciones en los gastos en salud significativas y sostenibles) y, finalmente, se calcula que el costo de este tipo de medicamentos es entre USD $\$ 50000$ a $\$ 250000$ por paciente y por año. Es por esto que IMS Health calcula que para el 2020 se facturen globalmente en ventas de biosimilares entre USD \$10 000 a USD \$25000 millones (5).

La diferencia del costo de producir un genérico (el cual se calcula entre 1 y 4 millones de dólares) con el de un biológico que logre comercializarse (solo el 10\% lo logra) ronda los 2000 millones de dólares; los biosimilares cuestan entre 100 y 250 millones de dólares para producirse y se toman de 7 a 8 años antes de su uso comercial, pero se calcula que su rentabilidad sea altísima luego de conocer que solo Remicade facturó globalmente para todas sus indicaciones 9200 millones y Humira 13000 millones de dólares en el 2014 (6). Lo anterior es cierto para los primeros biosimilares que compi$\tan$ con su originador porque, al ser el precio realmente su único argumento comercial, cada vez que entre uno nuevo al mercado, el valor miligramo a miligramo del originador y los antecesores se reducen para mantenerse competitivos $y$, por ende, los márgenes de intermediación y de retorno se disminuyen tanto que la inversión ya no va a ser rentable.

La EMA en el 2005 definió los conceptos de biológicamente similar o bioequivalente una vez que se estandarizaron las características básicas de los medicamentos biológicos (7):

- Biológico: es una medicina cuyo principio activo es una sustancia biológica (ADN recombinante, virus atenuado, derivados sanguíneos o plasmáticos, anticuerpos monoclonales, entre otros) producida o extraída de una fuente biológica física y químicamente caracterizada.

- Biológicos de nueva generación: son agentes de última tecnología cuyos blancos terapéuticos o dianas biológicas son las mismas del producto original, pero son elaborados de manera diferente e individual como productos nuevos que se someten a las pruebas de eficacia y seguridad convencionales (8).

- Biosimilar: es un medicamento desarrollado por otro laboratorio que sostiene que es similar al medicamento biológico original o de referencia (biológico aprobado y comercializado por las agencias regulatorias). Contiene el mismo componente activo, diseñado para ser usado contra la misma enfermedad por la misma vía de administración y a las mismas dosis que el medicamento de referencia.

Fue la EMA la primera agencia reguladora que acuñó el término "biosimilares" y desde el 2005 diseñó una vía para su regulación, habiendo aprobado hasta la actualidad $16 \mathrm{de}$ estos medicamentos (9). A nivel mundial, el primer biosimilar aprobado fue en abril de 2006, cuando la EMA autorizó la comercialización de un biosimilar de la somatropina (hormona recombinante de crecimiento humano) (10). 
El día "en que todo cambió" con respecto a la EII fue el 13 de septiembre de 2013 cuando la EMA dio el paso definitivo al aprobar el primer biosimilar del infliximab (IFX), el CT-P13 (Inflectra ${ }^{\circ}$ Remsima Celltrion Healthcare, Corea) para todas las indicaciones en las cuales el IFX estaba aprobado, incluidas: colitis ulcerativa moderada a severa en todas las edades, EC moderada a severa en todas las edades, al igual que EC fistulizante, AP, psoriasis, EA y AR (1).

La aprobación europea para estas indicaciones se basó en el reconocimiento de que el principal mecanismo de acción del IFX en las diferentes entidades es el mismo y que la información aportada por los estudios comprensivos no clínicos demostraron que las principales características fisicoquímicas y la actividad biológica del CT-P13 y el originador fueron comparables (11).

Con respecto a los estudios clínicos, la EMA se basó solamente en 2 estudios: el PLANETAS (en EA) y el PLANETRA (en AR) (12). El estudio PLANETAS, de fase I, se incluyeron 250 pacientes con EA y se demostró bioequivalencia entre CT-P13 y el IFX en términos de farmacocinética. La respuesta clínica a las semanas 14 y 30 fue del $63 \%$ y $71 \%$ para el CT-P13 frente al $65 \%$ y del $72 \%$ para Remicade. Los niveles de anticuerpos para infliximab (API) se presentaron en el $9 \%$ y $27 \%$ de los pacientes tratados con CT-P13 frente al $11 \%$ y el $23 \%$ de los tratados con Remicade a las semanas 14 y 30, respectivamente. Los eventos adversos incluyendo reacciones infusionales a la semana 30 fueron del $65 \%$ para el grupo de CT-P13 frente al 64\% para el de Remicade. Lo anterior permaneció equivalente hasta la semana 54 (13).

El estudio PLANETRA fue fase III con 606 pacientes con AR, los cuales se agruparon aleatoriamente para recibir CT-P13 (302 pacientes) frente a IFX (304 pacientes) $3 \mathrm{mg} /$ $\mathrm{kg}$ a las semanas $0,2,6$ y cada 8 semanas + metotrexato hasta la semana. El objetivo primario fue evaluar la equivalencia terapéutica con un intervalo de confianza (IC) del 95\% (14).

Luego de esta aprobación, el impacto económico no se hizo esperar ya que provocó que MSD, que comercializa el Remicade en Europa, disminuyera sus ingresos de US $\$ 2300$ millones en 2014 a US $\$ 1800$ millones en 2015, una vez fue introducido el biosimilar al mercado (6).

En mayo 2016, el SB2 (Flixabi', Samsung Bioepsis, Corea de Sur) fue el segundo biosimilar de IFX que la EMA autorizó para su comercialización en Europa para el tratamiento de colitis ulcerativa moderada a severa en todas las edades, EC moderada a severa en todas las edades, AP, psoriasis, EA, y AR. La aprobación se basó en datos clínicos de un estudio farmacocinético fase I aleatorizado que comparó SB2 y Remicade en 159 individuos sanos y en un estudio fase III doble ciego, multinacional, aleatorizado con grupos paralelos comparando SB2 al IFX de referencia en 584 pacientes con AR moderada a severa a pesar de la terapia con metotrexato (10).
El 9 de febrero de 2016, el comité asesor sobre artritis de la FDA se reunió para estudiar la aprobación de CT-P13 en Estados Unidos. La decisión de este comité, y siguiendo exactamente los lineamentos que llevaron a la EMA a tomar tal decisión, fue extrapolar los resultados de estos estudios y autorizar en bloque el uso de CT-P13 para todas las indicaciones en las cuales se usa el IFX y no de manera individual (indicación por indicación). Nunca se presentó un estudio clínico en colitis ulcerativa ni EC como soporte para la aprobación en dichas indicaciones. El 5 de abril de 2016, la FDA (con base en los mismos estudios en que se fundamentó la EMA, que demostraron biosimilaridad al compuesto original utilizando comparaciones de estructura, función, toxicidad animal, farmacocinética y farmacodinámica humanos, eficacia clínica, seguridad e inmunogenicidad con un cálculo de un IC 90\% y acogiendo la recomendación de dicho comité) aprobó el CT-P13 para todas las indicaciones de IFX (Remicade). El 12 de julio de 2016, el comité asesor sobre artritis de la FDA recomendó que el ABP 501, el biosimilar de adalimumab (ADA) (Humira) de Amgen (Thousand Oaks, California, Estados Unidos), fuera autorizado para ser usado y extrapolado en todas las indicaciones en los cuales el Humira se prescribe actualmente; con base en 2 estudios clínicos fase III, uno de AR y otro de psoriasis con placas (12). Hoy en día hay más de 20 biosimilares de IFX o ADA en desarrollo.

Aunque un clínico consideraría que la biosimilaridad debería estar más en función de eficacia y seguridad, para la FDA (y una vez más siguiendo los conceptos de la EMA) es más importante la demostración de la similitud analítica entre el producto propuesto y el de referencia más que establecer independientemente estas características anteriormente enumeradas. Inclusive en nuestro medio, lo anterior es ya una realidad cuando el 18 septiembre de 2014 el presidente Juan Manuel Santos Calderón sancionó la ley con el decreto número 1782 de 2014 del Ministerio de Salud y Protección Social (15), en la que se autoriza el uso de biosimilares en nuestro país a través de la ruta abreviada de la comparabilidad (4), no sin antes haber recibido presiones políticas por parte del gobierno estadounidense en un sentido contrario (ver la carta del vicepresidente Biden, Anexo 1).

Todo lo anterior es un tema de gran actualidad y aún mayor controversia por las implicaciones terapéuticas y económicas que esto conlleva ya que de antemano, de acuerdo con la evidencia aportada y debidamente aprobados, estos medicamentos no son menos eficaces ni menos seguros que los innovadores; por tanto, su argumentación y su mayor sustento para su comercialización es el ahorro de costos.

\section{DEFINICIONES}

Un biotecnológico es un fármaco cuyo principio activo es una sustancia biológica (ADN recombinante, virus atenuado, 
derivados sanguíneos o plasmáticos, anticuerpos monoclonales, entre otros) producido o extraído de una fuente biológica (proteínas derivadas del $\mathrm{ADN}$ ) física y químicamente caracterizado mediante procesos de hibridación y de recombinación requiriendo organismos vivos para su producción, con el objetivo de actuar sobre el sistema inmunitario (16). En el proceso inflamatorio intervienen diferentes grupos celulares y mediadores de la inflamación susceptibles de actuación terapéutica y son los llamados blancos terapéuticos (dianas biológicas).

La inhibición del factor de necrosis tumoral alfa (TNFa) se ha probado eficaz en el control de la inflamación moderada a grave en la EC y en la colitis ulcerativa, al igual que en otros desórdenes inflamatorios inmunes tales como EA, psoriasis y AR, lo cual ha causado una revolución en el manejo de estos pacientes, específicamente en la EII y en aquellos con compromiso fistulizante por EC ya que, antes del advenimiento de esta clase de medicamentos (hace tan solo 2 décadas) y después del estudio de Targan y colaboradores (17) no existía ninguna terapia tan efectiva (18), pues los esteroides inducen remisión, pero no están indicados para la fase de mantenimiento y los inmunomoduladores actúan para evitar recaídas, pero no en los brotes. La ventaja de los biológicos es que estos tienen tanto efectividad para inducir remisión como para el mantenimiento sin los efectos deletéreos de los esteroides.

La terapia anti-TNF- $\alpha$ ha tenido grandes logros y por primera vez en la EII moderada a grave se está logrando remisión profunda sostenida (remisión clínica, libre de esteroides, con biomarcadores negativos y cicatrización mucosa), lo que se traduce en prevención del daño estructural y reducción de hospitalización y cirugía; que finalmente resulta en menor morbimortalidad y mejoría en la calidad de vida para los pacientes (18-20).

En la actualidad disponemos en Colombia de 3 tipos de inhibidores o antagonistas del TNF- $\alpha$, del tipo anticuerpos monoclonales específicos (IFX, ADA y golimumab en colitis ulcerativa) y un biosimilar para IFX: CT-P13, Remsima (Celltrion, Corea del Sur) de uso clínico en el tratamiento de la EII; así mismo, se han desarrollado biosimilares para ADA: Amgen (Estados Unidos), que aún no está autorizado en nuestro país.

Este desarrollo es complejo, costoso y solo va a surgir si se cumplen 3 condiciones (9):

1. Una compañía farmacéutica encuentra un biológico interesante desde el punto de vista económico, cuya patente esté a punto de expirar.

2. Estructura todo un proceso de desarrollo, tomándose el riesgo y el costo de producir un biosimilar.

3. Que una agencia reguladora lo apruebe.

Existen diferentes posiciones entre las agencias reguladoras que incluso ni siquiera tienen un consenso de cómo se les debe denominar a estos fármacos: La FDA inicialmente los llamó productos de proteínas a continuación (follow-on protein products), aunque en sus últimas posiciones los denomina biosimilares; Salud Canadá (Health Canada) los llaman biológicos de entrada subsecuente (subsequent entry biologics); y la EMA los denomina biosimilares.

En nuestro medio es más conocido el término de biosimilar y, por tanto, es el que se utiliza en este artículo, aunque también se usa el de biocomparable, por ejemplo, en México (el país con el mayor número de personas hispanohablantes del mundo).

El problema con estas definiciones es el término similar ya que, antes de acuñar este concepto, el medicamento debe someterse a múltiples estudios in vitro tales como tolerabilidad, pureza, seguridad, bioequivalencia, efectividad, farmacocinética, entre otros; e in vivo a través de ensayos clínicos aleatorizados doble ciego controlados y con adecuado poder. Después de todo este proceso sí se puede acuñar el término de biosimilar (16). Pero para efectos de su comercialización, los laboratorios deben demostrar que su producto es similar al medicamento de referencia en términos de características fisicoquímicas, eventos adversos y eficacia clínica (21).

Los biosimilares no son considerados copias ni genéricos de los productos innovadores, ya que los genéricos tradicionales se consideran terapéutica y estructuralmente idénticos a la fórmula química original e implican que la sustancia activa sea idéntica al medicamento de referencia. La mayoría de los fármacos sintéticos tiene un bajo peso molecular de solo unos cientos de Daltons (Da) (el ácido acetilsalicílico tiene $180 \mathrm{Da}$ con 21 átomos y el omeprazol tiene $345 \mathrm{Da}$ con 17 átomos de carbono). Este no es el caso de los biosimilares, ya que el principio activo de un biológico es una estructura compleja tridimensional con alto peso molecular (IFX tiene $149000 \mathrm{Da}$ y ADA $150000 \mathrm{Da}$, ambos con >20000 átomos), cuya heterogeneidad, dependencia en la producción de cultivos celulares y procesos de manufactura altamente sofisticados tales como modificaciones postranslacionales (la glicosilación, fosforilación, desamidación, oxigenación y pegilación) los hacen completamente diferentes a una molécula individual poco compleja de bajo peso y técnicas tradicionales de manufactura, que es la forma como se presentan los medicamentos convencionales; además de que estos tienen la capacidad de inducir una respuesta inmunológica (Figura 1). Aunque la estructura global del biológico sea conocida, el proceso de su manufactura es desconocida ya que esto hace parte del secreto de propiedad intelectual; por tanto, los métodos analíticos actuales no pueden caracterizar estas moléculas complejas con las suficientes garantías para confirmar bioequivalencia estructural con las moléculas de referencia (22-24), y así se pudiera, la equivalencia clínica solo podría ser probada en estudios clínicos. 


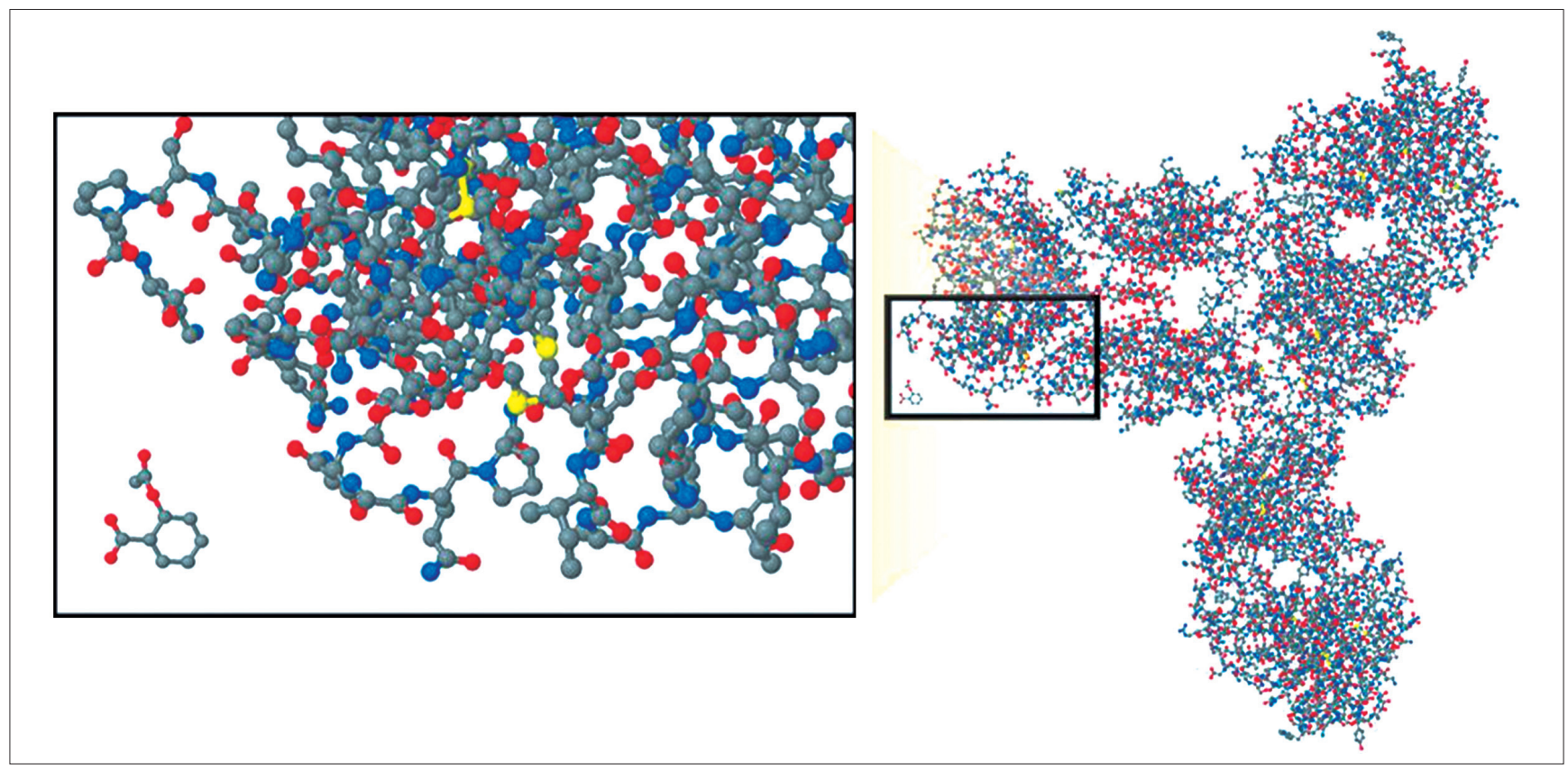

Figura 1. Comparación entre un anticuerpo monoclonal biológico y una molécula de ácido acetilsalicílico. Tomado de: Kozlowski S, Woodcock J, Midthun K, et al. Developing the nation's biosimilars program. N Engl J Med. 2011;365(5):385-8.

Cuando se analizó fisicoquímicamente el CT-P13 frente al originador, su estructura aminoproteica es idéntica con la excepción de una glicina C-terminal. Las estructuras de un orden mayor, al igual que la pureza monomérica, son similares. Se demostró comparabilidad en la actividad biológica entre ambos productos al neutralizar el TNF in vitro de manera similar (12).

Se hicieron estudios en voluntarios sanos que, de acuerdo con la FDA, son considerados los más sensibles para encontrar diferencias entre biosimilares y los productos de referencia. Se diseñó un estudio doble ciego de 3 brazos con grupos paralelos para determinar las propiedades del PK del CT-P13 (70 pacientes) y 2 formulaciones de IFX (Remicade ${ }^{\circledR}$ europeo: 71 pacientes, y Remicade ${ }^{\circledast}$ norteamericano: 70 pacientes) luego de una única infusión de $5 \mathrm{mg} / \mathrm{k}$ en sujetos sanos. Se midieron los niveles séricos del PK en diferentes momentos tales como antes de la infusión, inmediatamente después de esta y hasta el día 57 después de infusión. Las 3 formulaciones fueron esencialmente equivalentes en términos de concentración máxima $\left(\mathrm{C}_{\text {máx }}\right)$ de IFX, área bajo la curva del tiempo de concentraciones séricas, y no hubo diferencias de eventos adversos entre los 211 pacientes evaluados (25). La posición de Health Canada es aún más rigurosa que la FDA, pues entre sus políticas utiliza términos supremamente puntuales y delicados como son: intercambiabilidad, extrapolación y substitución; las guías canadienses enumeran que solamente se puede considerar un biosimilar cuando ya exista un biológico original aprobado (algo también mencionado por la FDA) y, entre otras afirmaciones, dichas guías son puntuales en lo siguiente (26):

- El biosimilar se puede considerar "similar" al original luego de haber sido sometido y superado todas las pruebas de "biosimilitud" tales como estructura bioquímica similar, propiedades farmacocinéticas y farmacodinámicas similares y la comprobación de esta similitud en estudios clínicos.

- Los biosimilares, aunque son biológicos, no son biológicos genéricos y su autorización no los declara farmacológicamente con equivalencia terapéutica a los biológicos de referencia.

- Un biosimilar se debe considerar un nuevo biológico y se debe regular igual a este. Un biosimilar no se debe escudar en una indicación del biológico de referencia para ser extrapolado en otras indicaciones, aunque ya Health Canada autorizó la extrapolación de CT-P13 para todas las indicaciones del originador el 10 de octubre de 2016 (27).

- Un biosimilar no se debe etiquetar como intercambiable por un biológico originador por el profesional de salud.

- Un biológico originador no puede ser sustituido por un biosimilar en el sitio de dispensación de estos medicamentos.

De acuerdo con un estudio holandés, los biológicos son responsables del $64 \%$ del costo total del manejo de la EC y del $31 \%$ en el manejo de la colitis ulcerativa. Se estima que 
el valor de los biosimilares miligramo a miligramo es entre un $25 \%$ a un $40 \%$ menor que el costo de los biológicos (9). Todo lo anterior ha despertado un debate en temas tales como la seguridad y la preocupación sobre bioequivalencia con respecto al potencial de inmunogenicidad y de eventos adversos inmunológicos.

Existen 3 términos que en el contexto de los biosimilares se deben entender: extrapolación, sustitución e intercambiabilidad (28).

\section{Extrapolación}

Se refiere a la autorización por parte de una agencia reguladora de un biosimilar ya aprobado para ser usado en una enfermedad en la cual no ha sido estudiado clínicamente. Esto es lo que precisamente ha pasado con el CT-P13, y es que con estudios extensos comparativos in vitro, pero ninguno clínico en EII y solo 2 de comparación cabeza a cabeza en pacientes con AR y EA, bajo la premisa de que una "alta similitud" en los estudios predice resultados clínicos "altamente similares"; las diferentes agencias reguladoras (EMA, FDA, Health Canada e Instituto Nacional de Vigilancia de Medicamentos y Alimentos -INVIMA-) los han autorizado para ser usados en todas las otras indicaciones (extrapolación) que el original (Remicade ${ }^{\circ}$. El principal argumento para aceptar la extrapolación es que la alta "similitud" analítica es la base científica más robusta para comparar biológicos de orígenes independientes (9). Pero cuando se analizan los estudios más detalladamente, se encuentra que el estudio PLANETRA, de no inferioridad fase 3 en AR de 606 pacientes con Remicade como comparador, diseñado como un estudio de equivalencia y el IC del $95 \%$ para la diferencia terapéutica entre ambas moléculas para el objetivo primario fue $-6 \%$ al $10 \%$, lo cual cae en el margen de equivalencia seleccionado $+/-15 \%$ y reportando una diferencia frente a este de un $2 \%$ de efectividad y con unos niveles de inmunogenicidad similares entre el CT-P13 y el originador. Cabe anotar que los pacientes en este estudio recibieron conjuntamente metotrexato, el cual actúa como un inmunomodulador, lo que evita o retrasa la formación de anticuerpos antiinfliximab (ATI) y, por ende, no permite que se aprecien temprana o fácilmente diferencias en inmunogenicidad entre ambos productos. Además, se escogió la enfermedad menos sensible como modelo clínico para detectar una diferencia potencial en eficacia entre el biosimilar y el originador, que en otras circunstancias si sería evidente. En otras palabras, la efectividad en similitud demostrada por el CT-P13 y el Remicade para la AR no excluye la posibilidad de una diferencia clínicamente significativa en efectividad entre ambas moléculas si se aplican en un modelo mucho más sensible, tal como la EII (29).
Es por este criterio de extrapolación por el cual Health Canada inicialmente autorizó el CT-P13 para su uso en AP y en placas prosísticas, pero no en EII; lo cual ya recientemente modificó, siendo aprobado para todas las indicaciones del Remicade en la EII (27).

En el estudio PLANETAS, fase I, en el que se incluyeron 250 pacientes con EA, se demostró bioequivalencia entre CT-P13 y el Remicade en términos de farmacocinética. Esta metodología no puede ser aceptable para las agencias reguladoras al evaluar una molécula innovadora. Por tanto, el concepto de equivalencia real es cuestionable (26).

Se requiere más investigación cuando los datos de eficacia y seguridad se extrajeron de estudios clínicos de pacientes con $\mathrm{AR}$, porque en esta entidad el mecanismo de acción del anti-TNF es básicamente la neutralización del TNF; en cambio, en la EII estos medicamentos actúan disminuyendo probablemente la producción de citoquinas que inducen la apoptosis de los monocitos y linfocitos activados (28), promueven macrófagos M2 cicatrizadores de mucosa, al igual que la citotoxicidad mediada por complemento y por células (30). Además, la tasa de aclaramiento del IFX es $40 \%$ mayor en pacientes con EII que con EA.

\section{Sustitución}

Sustitución automática: entregar un medicamento por otro en la farmacia sin consultar al médico prescriptor.

\section{Intercambiabilidad}

De acuerdo con la FDA, un medicamento biosimilar puede ser designado como intercambiable si se espera que produzca el mismo resultado clínico que el producto de referencia en un determinado paciente y la alternancia repetida entre el medicamento biosimilar y el producto de referencia no supone riesgos de seguridad ni eficacia $(21,31,32)$.

La EMA no expresa recomendaciones sobre intercambiabilidad y las pautas de sustitución quedan a criterio de cada estado miembro de la Unión Europea (UE) (31-33).

Intercambio por motivos médicos: intercambio de un fármaco por otro por razones clínicas en un paciente con una respuesta inadecuada a un tratamiento o que presenta un evento adverso intolerable.

Intercambio por motivos no médicos: intercambio de un fármaco por otro en un paciente que está recibiendo un tratamiento adecuado y bien tolerado; esta situación se da debido a razones administrativas o de pagador.

El problema es poder entender la respuesta inmunológica en un contexto de intercambios múltiples porque solo entenderla en un determinado medicamento biológico ya es complicado y no completamente explicable ni entendible; además, es posible que no se detecten reacciones 
inmunogénicas hasta tiempo después de iniciarse su administración; por tanto, puede ser difícil entender y rastrear potenciales reacciones inmunogénicas después de realizar múltiples cambios. (33-36).

La EMA es supremamente estricta en la farmacovigilancia de los biosimilares, pues exige un plan detallado del manejo de riesgos; este plan se considera inclusive más importante si la extrapolación es aceptada (9).

La intercambiabilidad es a lo que se refieren un artículo y su comentario $(37,38)$ ya que se investigó la comparabilidad inmunogénica entre Remicade y CT-P13, al saber que todos los anticuerpos monoclonales generan respuesta inmunológica contra el medicamento, y fue por esta razón que se quiso examinar si los anticuerpos producidos por el tratamiento con Remicade en pacientes con EII también reaccionan de manera cruzada y reconocen el CT-P13 en un grado similar. Si lo anterior fuera cierto, se podría pensar en intercambiar en un paciente de manera libre entre el agente innovador y el similar sin perder la eficacia y la seguridad comprobadas del primero a un costo hasta de un $40 \%$ menor. Lo anterior sugiere que en un individuo que haya generado títulos altos de ATI y que presente alguna reacción adversa a la infusión o pérdida de la respuesta no se justifica utilizar el otro "agente" IFX ya que con alta probabilidad presentará el mismo evento adverso.

En contraste, en un individuo con ATI negativos se podrían intercambiar libremente ambos agentes sin provocar algún tipo de reacción inmunológica adversa porque, de acuerdo con lo anterior, los anticuerpos anti-Remicade de pacientes con EII reconocen y funcionalmente inhiben el CT-P13 en un grado parecido, lo que sugiere una inmunogenicidad similar al compartir estos 2 agentes IFX epítopos inmunodominantes; pero es importante hacer énfasis en que antes de estandarizar este abordaje como para uso rutinario, se deben hacer estudios clínicos en los que se examine el suero de pacientes tratados con CT-P13 y que desarrollen ATI antiCT-P13 para evaluar su reactividad cruzada con IFX. Cabe resaltar que, aunque hay anticuerpos compartidos por el Remicade y CT-P13, puede haber otros exclusivos para cada uno pues son similares, no iguales $(37,38)$.

En este estudio se evidenció que los anticuerpos antiadalimumab (AAA) en pacientes tratados con EII no presentaron reacción cruzada hacia CT-P13 o Remicade; por tanto, se infiere que si un paciente presenta reacción adversa o falta de respuesta debido a AAA, se podría cambiar este por CT-P13 o Remicade indistintamente con una buena respuesta; aunque también se ha documentado que un paciente que tenga los ATI altos tendrá una probabilidad de generar tempranamente AAA cuando sea expuesto al ADA.

En 3 estudios observacionales de cohortes en EII (3941) que evaluaron el CT-P13 y el IFX, reportaron datos sobre la efectividad y seguridad al intercambiar entre el medicamento de referencia y el biosimilar. Aunque a modo de crítica sobre estos estudios, cabe anotar que todos los pacientes estaban en remisión antes del intercambio del medicamento de referencia al CT-P13, con un número de muestra pequeño, sin grupos comparadores en los que se hubiera continuado con el originador y alta heterogeneidad en cuanto al momento del intercambio, inclusive algunos de los pacientes no tenían tratamiento previo con el IFX y su protocolo de inducción se realizó con el CT-P13.

En Noruega se diseñó un estudio de cohortes prospectivo observacional que evaluó 78 pacientes (46 con EC: $28 \%$ con exposición previa a biológicos, y 32 con colitis ulcerativa: $16 \%$ expuestos previamente a biológicos) que recibieron CT-P13 a una dosis de $5 \mathrm{mg} / \mathrm{k}$ en las semanas 0,2 y 6 . A la semana 14 se encontró un $79 \%$ de pacientes con colitis ulcerativa y $56 \%$ con EC en remisión clínica con biomarcadores negativos (proteína C-reactiva $[\mathrm{PCR}]$ y calprotectina) (42).

Un estudio multicéntrico prospectivo observacional recientemente publicado que involucra la experiencia de toda Hungría evaluó la eficacia y seguridad a largo plazo. Se documentaron remisión, respuesta clínica y bioquímica a las semanas 14,30 y 54 . Ningún paciente había recibido IFX (originador) 12 meses antes. De los 291 pacientes reclutados inicialmente ( 184 con EC y 107 con colitis ulcerativa), el $25 \%$ (EC) y $14 \%$ (colitis ulcerativa) estuvieron expuestos previamente al uso anti-TNF, y $60 \%$ (EC) y $52 \%$ (colitis ulcerativa) usaron inmunosupresores concomitantes; al cabo de las 54 semanas se pudieron evaluar $100 \mathrm{de}$ estos pacientes. En este estudio, en EC hubo una respuesta clínica a las semanas: 14 (83\%), $30(77 \%)$ y 54 (58\%); y una remisión clínica a las semanas 14 (55\%), 30 (57\%) y $54(47 \%)$. Con respecto a la colitis ulcerativa, unas tasas de respuesta clínica a las semanas 14 (78\%), 30 (69\%) y 54 (64\%) y una remisión clínica a las semanas 14 (59\%), 30 $(46 \%)$ y 54 (53\%); con eventos adversos consistentes en $21(6,6 \%)$ reacciones infusionales, 23 (7,9\%) infecciones y 1 muerte. Por tanto, estos autores concluyen que el CT-P13 es seguro y efectivo tanto para inducir remisión como para su mantenimiento en pacientes con colitis ulcerativa y EC, aunque las tasas de respuesta y remisión, tal y como se tiene conocimiento, son más bajas en los individuos previamente expuestos a biológicos en el pasado (43).

Con respecto a la bioseguridad, en Hungría se realizó un estudio sobre datos de la vida real con 384 pacientes consecutivos (253 con EC y 131 con colitis ulcerativa) tratados con CT-P13, de los cuales el $24,5 \%$ con EC y el $14 \%$ con colitis ulcerativa habían recibido previamente terapia antiTNF. 21 pacientes $(7,2 \%)$ presentaron reacciones infusionales durante la inducción y la fase de mantenimiento, 13 de los cuales habían recibido IFX en el pasado. Las reacciones más frecuentes fueron alérgicas en el 44,4\%, disnea 
y dolor torácico en el $27,7 \%$, rubor en el $27,7 \%$, tos en $22,2 \%$, artralgia en $11,1 \%$ y cefalea en $11,1 \%$. Hubo necesidad de suspender/intercambiar el CT-P13 en el 62\% (237 pacientes), por lo que se cambió a $\mathrm{ADA}$ en 146 pacientes $(38,1 \%)$ y, a pesar de lo anterior, se continuó en 82 pacientes $(21,4 \%)$ con CT-P13 asociado con intervención suplementaria (antihistamínicos/esteroides/infusión lenta). Se documentó que los factores predictivos de mal pronóstico para el cambio fueron la exposición previa a anti-TNF y tener ATI, pero a su vez el uso de azatioprina (AZA) (limítrofe) y la normalización de la PCR a la semana 14 fueron considerados como factores protectores. En conclusión, los autores de este estudio consideraron el uso del CT-P13 como seguro, con una baja incidencia de reacciones infusionales adversas (44).

El estudio Nor-switch, también con datos de la vida real, se diseñó como un estudio observacional prospectivo aleatorizado doble ciego de grupos paralelos con estabilidad clínica mayor a 6 meses recibiendo IFX; se comparó el CT-P13 frente al IFX en 6 enfermedades inflamatorias inmunológicamente mediadas (IMID): EC, colitis ulcerativa, EA, AR, psoriasis y AP. A la mitad (240) se les intercambió (switch) por CT-P13 y al otro 50\% (240) se les siguió la terapia estándar con IFX. El objetivo primario era evaluar el empeoramiento de la enfermedad después de 52 semanas. De manera global, recayeron 53 pacientes $(26,2 \%)$ en el grupo de IFX frente a 61 pacientes $(29,6 \%)$ tratados con CT-P13 con un IC 95\% (-12,7\%-3,9\%), lo cual muestra que no hay diferencias clínicamente significativas al cabo del año; pero cuando se evalúan los subgrupos, la EC recayó más frecuentemente cuando se usó el CT-P13: 23 pacientes $(36,5 \%)$ frente a 14 pacientes $(21,2 \%)$ tratados con IFX, con un IC (-29,3\% a -7\%), lo cual es clínicamente significativo (45).

Cabe aclarar que este estudio hay que analizarlo con cuidado ya que engloba múltiples indicaciones, falta claridad en las definiciones, incluidos los criterios de inclusión y exclusión, objetivos terapéuticos, impacto y uso de medicación concomitante. Todos estos factores crean confusión y, por ende, no le proporcionan el poder estadístico robusto como para soportar una interpretación rigurosa (46).

Se evaluó la capacidad de cicatrización de la mucosa (CM) en colitis ulcerativa luego de la inducción con CT-P13 en 63 pacientes resistentes al tratamiento convencional o que fueran esteroideo-refractarios, esteroideodependientes o que necesitaran rescate precoz debido a una colitis aguda tóxica. A las 14 semanas se evaluó la respuesta clínica $(82,5 \%)$, remisión libre de esteroides $(47,6 \%)$, cicatrización mucosa libre de esteroides $(47,6 \%)$ y la cicatrización completa de mucosa (27\%); además de determinar que los niveles valle de IFX se correlacionaron con la CM. De acuerdo con estos autores, la CM se logra en 2/3 partes de los pacientes con colitis ulcerativa al final de la dosis de inducción (semana 14) con CT-P13 (47).

El único estudio que comparó la costo-efectividad entre el medicamento originador y su biosimilar en pacientes con EC luminal lo hizo el grupo húngaro, además de comparar la costo-efectividad entre los diferentes biológicos usados en Europa; se documentaron los costos en 9 países europeos (Bélgica, Francia, Alemania, Hungría, Italia, Holanda, España, Suecia e Inglaterra). Se diseñó un modelo probabilístico de Markov para analizar la costo-efectividad de secuencias de tratamiento seleccionadas, comparadas con el estándar de manejo en pacientes con EC luminal activa moderada a severa que no respondieran al tratamiento convencional. El uso del biosimilar frente al tratamiento convencional se incrementó desde los $€ 35170$ en Hungría hasta los €71 624 en Suecia. Cuando esto se comparó con el uso del Remicade frente al tratamiento convencional, los costos se incrementaron desde los $€ 69000$ en Hungría hasta los $€ 135000$ en Alemania. La inclusión de biológicos adicionales a las secuencias de tratamiento lógicamente incrementaron los costos por año por paciente, pero siempre fue más barato cuando, independientemente de la secuencia escogida (CT-P13-, Remicade, ADA y/o vedolizumab [VDZ]), en algún momento se usó el biosimilar. Por tanto, y de acuerdo con el concepto de los autores, con base en los resultados de costo-efectividad, concluyeron que el CT-P13 se puede recomendar como una primera línea de tratamiento en EC que no responda al tratamiento convencional. La secuencia CT-P13, ADA, VDZ se identificó como la más costo-efectiva y, finalmente, el uso del CT-P13 es una alternativa costo-efectiva al producto originador para el tratamiento de adultos con EC luminal y esto puede contribuir a aumentar el acceso a estos productos (48).

Existe un estudio holandés en el que, a través de una proyección matemática, se simuló la realidad de costos después de 5 años de introducción de los biosimilares al mercado con base en una reducción gradual de los biosimilares del $60 \%$, del originador del $50 \%$ y un incremento anual de la prescripción de anti-TNF- $\alpha$ del $1 \%$. Los resultados fueron un ahorro promedio en costos totales a 5 años de $€ 9850$ por paciente con EC, de $€ 2250$ por paciente con colitis ulcerativa; y un ahorro global de $€ 493$ millones en los costos por terapia anti-TNF- $\alpha$ en los Países Bajos (49).

Finalmente, quiero plasmar las posiciones de las diferentes asociaciones de gastroenterología sobre la introducción de biosimilares como opciones terapéuticas:

La Asociación Canadiense de Gastroenterología está en contra de la intercambiabilidad del agente de referencia y las sustituciones automáticas a nivel de farmacia, basado en una motivación de costos (26).

La Sociedad Española de Patología Digestiva está en contra de la extrapolación de los resultados del estudio 
PLANETRA a la población que padece EII, debido a la variabilidad biológica a través de los diferentes estados de la enfermedad; en cambio, aboga por estudios clínicos del biosimilar para las indicaciones individuales, como se les exigió a los agentes biológicos de referencia (16).

La posición del Grupo Italiano de EII recomienda altamente que se provea evidencia de la eficacia y seguridad del biosimilar en el marco de la EII antes de su comercialización. También están en contra de la intercambiabilidad de los biosimilares y no apoyan la extrapolación a través de las indicaciones clínicas, en particular debido a la heterogeneidad de la presentación y el curso clínico de los pacientes con EII (50).

La Organización Europea de Crohn y Colitis (ECCO) en el 2013 hizo énfasis en la necesidad de mayores estudios clínicos específicos en EII con el poder estadístico de biosimilares suficiente para detectar las diferencias no solo en eficacia en el corto plazo sino seguridad e inmunogenicidad en el largo plazo (8). Aunque en el 2017 y después de una reunión de consenso de expertos realizada el 15 de octubre de 2016 en Viena, Austria (51), se elaboró un nuevo documento al respecto en el que su posición ha cambiado de manera importante y es mucho más abierta con conceptos tales como:

- Los estudios clínicos de equivalencia para la indicación de prescripción más sensible pueden proveer las bases para la extrapolación; por ende, la información del uso de biosimilares en otra indicación sensible puede ser utilizada para tratar la EII.

- Solo estudios observacionales y con registros serios a largo plazo en un escenario de la vida real demostrarán la seguridad de estos medicamentos.

- Los eventos adversos y la pérdida de respuesta debida a la inmunogenicidad de un biológico originador no se espera que sean evitados con el uso de su biosimilar.

- El cambio o switch único entre el originador y el biosimilar es aceptada, aunque aún falta evidencia clínica con respecto al cambio o switch reverso, intercambiabilidad múltiple o intercambiabilidad entre biosimilares.

- La intercambiabilidad entre el originador y el biosimilar se puede hacer una vez haya un conocimiento amplio y claridad frente a esta entre todos los actores involucrados (prescriptor, farmaceuta, enfermero, paciente y pagador), ya que en una encuesta reciente realizada a 1181 pacientes por la Federación Europea de Asociaciones de Crohn y Colitis, solo el 38\% sabía sobre estos; pero más importante aún, el 55,9\% de los que contestaron piensa que el menor costo de estos medicamentos no debe primar sobre la seguridad y eficacia terapéutica.

La posición de la Fundación de Crohn y Colitis de América (CCFA) sobre biosimilares enfatiza la necesidad de tomar una decisión compartida y transparente en contra de las sustituciones automáticas y permitir a los proveedores determinar si la sustitución de un biosimilar frente a un producto de referencia es aceptable. Adicionalmente, se deben tener precauciones en contra de intercambiabilidad hasta que haya mayores pruebas basadas en evidencia sobre seguridad, eficacia e inmunogenicidad (52).

La Sociedad Británica de Gastroenterología publicó unas guías en las que apoya el inicio de terapia con CT-P13. Además, aprobaron el cambio de pacientes estables con el IFX originador al biosimilar, pero no recomiendan la sustitución automática en la farmacia sin el conocimiento del médico tratante (53).

Todas las sociedades recomiendan unos números de identificación únicos y nombre en concordancia con los nombres internacionales no patentados, como lo exige la Organización Mundial de la Salud (OMS) para prevenir la confusión por parte del paciente, el proveedor y la farmacia cuando se hace referencia a un medicamento originador frente a un biosimilar. El grupo de expertos de la OMS sobre nombres internacionales no propietarios (INN) propuso en el 2014 un sistema de 2 componentes para identificar a los biosimilares, siendo el primer componente del nombre el INN y la segunda parte un código único (identificador biológico) que consiste en 4 letras asignadas de manera aleatoria (54).

En Europa, un biosimilar comparte el mismo INN que su originador. Esta decisión se tomó en octubre de 2013 con el objetivo de mantener una relación cercana con su originador ya que, si se asignaba un INN diferente, esto crearía desconfianza tanto en el público como en el personal sanitario para el uso de estos productos (55).

Recientemente, en enero de 2017, la FDA publicó unas guías con respecto al nombramiento de agentes biosimilares no patentados para evitar la confusión y asumir de manera inadvertida su intercambiabilidad. Los nombres incluirían sufijos sin ningún significado, compuestos de 4 letras. De acuerdo con esta regla, el Inflectra llevaría el sufijo -dyyb para diferenciarse (infliximab-dyyb). De manera importante, esta regla también afecta agentes antiguamente aprobados tales como el Remicade ${ }^{\varpi}$, cuyo nombre oficial sería "infliximab-hjmt"; lo cual evita percepciones imprecisas de que un biosimilar difiere de manera clínicamente significativa o es inferior para las indicaciones prescritas $(31,32)$.

\section{CONCLUSIÓN}

Si la bioequivalencia e intercambiabilidad se pueden demostrar con los biosimilares y la molécula innovadora en pacientes con EII, con base en un ahorro en costos, lo que a su vez abriría una nueva puerta para el inicio más temprano de estos medicamentos en pacientes que los requieran o combinarlos con otras moléculas (VDZ) que hasta el momento no se hace por los altos costos (28), pero el dilema central tanto para pacientes, prescriptores y aseguradores es hasta qué grado un 
biosimilar A es intercambiable con el originador A, bajo cuáles circunstancias y a cuáles pacientes. Pero para complicar más el asunto, ¿con la llegada de nuevos biosimilares B, C, D, $\mathrm{F}$, etc. del originador $\mathrm{A}$, estos también se podrán intercambiar libremente entre ellos o con el producto de referencia con el mismo perfil tanto de seguridad como de eficacia? Sin contar cuando, aparte del originador A, también haya biosimilares para los originadores B o C (Figuras 2 y 3 ).

En una mesa redonda sobre biosimilares que se llevó a cabo el 12 de enero de 2016 en Bruselas, entre reguladores europeos y las sociedades médicas concluyeron que el entendimiento sobre el concepto de biosimilares entre los prescriptores y los reguladores es diferente, aunque esta situación está cambiando, luego de conocerse una mayor información de estos productos, pero sobre todo porque la experiencia de la vida real en países en donde se ha masificado su consumo, incluida la intercambiabilidad, ha sido positiva. Por tanto, más que una amenaza, los biosimilares se deben enfocar como una oportunidad, lo que debe promover una comunicación multidireccional entre todos los actores del proceso para definir intercambiabilidad, farmacovigilancia, trazabilidad, entre otros (56).

Pero, en definitiva, los biosimilares llegaron para quedarse, lo cual es una realidad con la cual se debe convivir $y$, aunque la evidencia existente apoya la biosimilaridad, la intercambiabilidad aún no tiene evidencia y por este motivo la FDA está trabajando en un borrador guía sobre el tema. Los datos, aún no concluyentes, que hay hasta el momento son de un único switch o cambio bajo supervisión médica, lo cual es diferente a intercambiabilidad. El

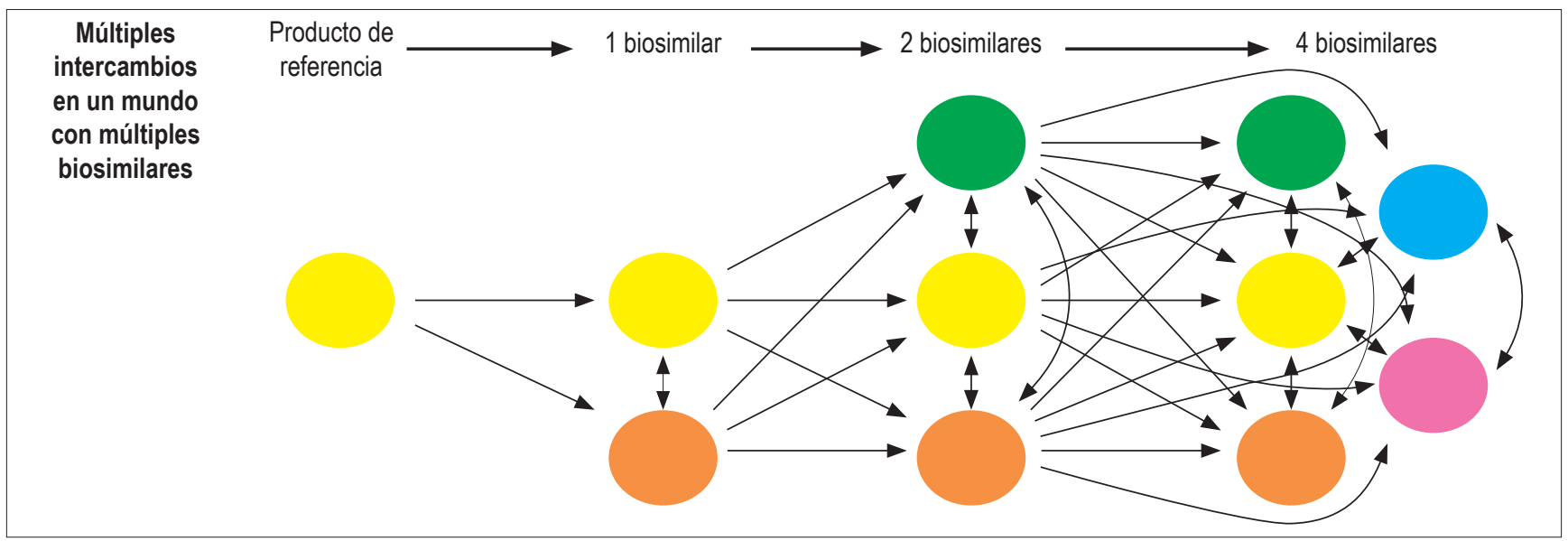

Figura 2. Esquema de múltiples intercambios entre biosimilares. Tomado de: Faccin F et al. Expert Opin Biol Ther. 2016;16(12):1445-53.

A Diseños de estudios para medicamentos originadores y biosimilares

Estudio de transición

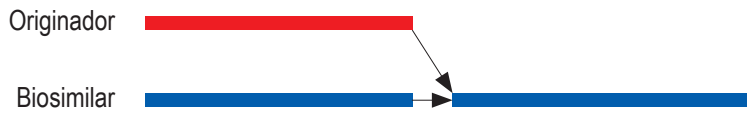

Estudio de cambio único

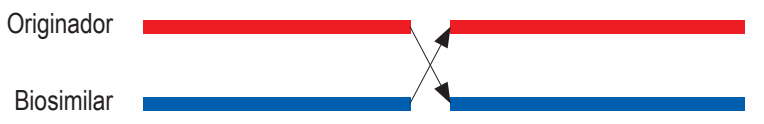

Estudio de cambio múltiple (para demostrar intercambiabilidad)

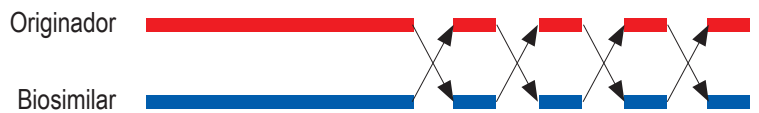

B

Escenario de la vida real en la práctica clínica

\section{Práctica clínica}

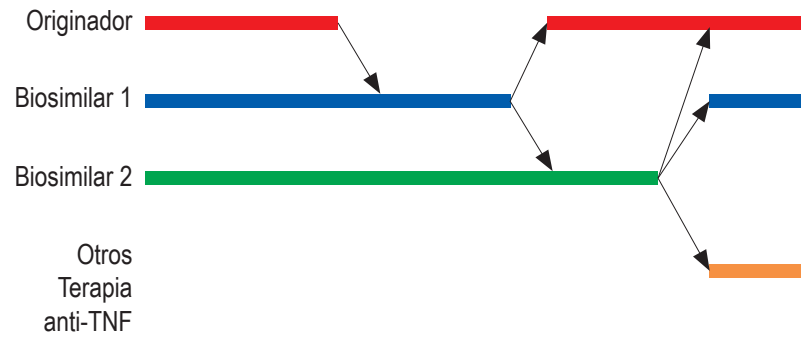

Figura 3. Escenarios esquemáticos de la vida real de múltiples intercambios entre biosimilares y originador. Tomado de: Faccin F et al. Expert Opin Biol Ther. 2016;16(12):1445-53. 
principal argumento para la producción y comercialización de estos nuevos anti-TNF en el manejo de las IMID fue el de un ahorro en costos hasta del $40 \%$ (lo cual, en la realidad y desde 2013, año en que se comercializa en la UE, el descuento frente al Remicade ha sido entre el $10 \%$ y $30 \%$, con un promedio del $25 \% \mathrm{y}$, probablemente, en nuestro país es inclusive menor) (12); por tanto, el conocimiento necesario para saber usarlos óptimamente es la mejor herramienta de la que se dispone ante los entes reguladores, los agentes pagadores y, finalmente, ante los pacientes que, en definitiva, son nuestra razón de ser.

\section{Agradecimientos}

Al Doctor Harold Perdomo, médico inmunólogo (Medical Scientific Liaison, Abbvie. Bogotá, Colombia) y a la Doctora Natali Serra-Bonett (regional Lead Biotherapeutics LATAM Global Medical Affairs, Abbvie. Ciudad de México, México).

\section{ANEX0 1}

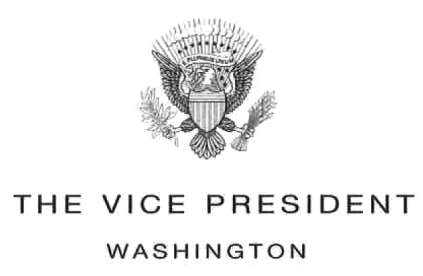

September 8, 2014

His Excellency

Juan Manuel Santos Calderón

President of the Republic of Colombia

Bogotá

Dear Mr. President:

A letter offers poor substitute for missing your August 7 inauguration, but I wanted to congratulate you all the same and to express my gratitude for your incredible hospitality during my visit to Bogotá so soon after your re-election. You assume a second mandate at a time of great promise for Colombia - within grasp of a historic peace, with limitless economic prospects, and ascending in diplomatic reach. Such opportunities, once unthinkable, are a tribute to your leadership and a testament to the perseverance, sacrifice, and undying optimism of the Colombian people. As Colombia embarks upon its ambitions, rest assured that the United States remains its most steadfast supporter and staunchest advocate. That is why President Obama and I would like to deepen our partnership in the months and years to come.

The future of the U.S.-Colombia relationship will be defined by our ability to build upon our bilateral trade agreement to promote our shared prosperity; broaden the scope of our bilateral relationship to seize opportunities and address shared challenges; and parlay our longstanding security cooperation into a strategic partnership with positive regional and global implications. We are already making quick progress, but as you told me in June, now is not the time to rest in our efforts to do even more together. I believe you would also agree that as we strive to realize the potential of our bilateral relationship we should also perfect the efforts currently underway. In that regard, I would like to outline some of the key aspects of our bilateral relationship as we have discussed them over the years. 
First, Colombia's prosperity is in the national security interest of the United States. The U.S.Colombia Trade Promotion Agreement (TPA), which entered into force in May 2012, solidified our trade relationship and currently represents roughly $\$ 40$ billion per year in two-way trade. The TPA has provided new opportunities to American and Colombian workers, while providing environmental and labor safeguards, and its benefits will only grow as Colombia continues to unlock its economic potential. At the same time, Colombia's long tradition of sound democratic and economic policies provide a model for other emerging economies, which is one of the reasons we are working with you to advance your timely accession to the Organization for Economic Cooperation and Development.

The TPA was just the beginning. We are promoting our people-to-people ties, most recently by extending reciprocal concession of visas from five to 10 years. With regard to the priorities outlined in your inaugural speech on education and technology, we are working together to expand English teacher training to public schools in Colombia via teacher colleges and increasing private sector support for two-way student exchanges as part of President Obama's 100,000 Strong in the Americas Initiative. I also applaud your efforts to establish strategic partnerships on education with U.S. states, like Massachusetts, and stand ready to support additional outreach by your government to U.S. states. On July 31, our governments kicked off an Information and Communications Technology Steering group where education, innovation, and development figured prominently on the agenda. There is still much more we can do and I look forward to our continued collaboration in these areas.

In order to deepen and expand our bilateral economic cooperation, we should maintain our focus on pressing matters of consequence to our trade agenda. Among these are the implementation of Colombia's intellectual property rights commitments and sustained progress on the Colombia Labor Action Plan. We remain concerned about the truck scrappage decree, which is hurting our bilateral trade (and arguably Colombian air quality, as well); new restrictions on the import of ethanol; and the biologics decree, which the World Health Organization and U.S. experts believe could put health and safety at risk. Ultimately, such discussions and policy differences should be understood as the result of our shared success and our desire to build on that success, and I am confident our governments can find a way to address these issues.

Second, our bilateral agenda today is increasingly characterized by issues beyond our borders in areas like energy and citizen security. We agreed on June 18 to redouble our efforts to promote energy security in the Caribbean. As previewed with you, I used my stop in the Dominican Republic to lay out a roadmap for the international community to support the Caribbean to secure its energy future. Toward this end, our Special Envoy and Coordinator for International Energy Affairs Amos Hochstein is working closely with your Minister of Energy on joint strategies to implement our commitments to energy security in the Caribbean. I have also instructed the Departments of State and Energy to work with our partners to organize a conference that would include the participation of Caribbean governments, multilateral financial institutions, donors, and private sector representatives. I hope we can count on representation from your government at the upcoming conference.

Third, Colombia has much to offer the world on security matters. Particularly as the conflict winds down and Colombia moves toward a more sustainable security strategy, organizations like the United Nations and the North Atlantic Treaty Organization (NATO) would benefit from your increased involvement in peacekeeping operations. Today's security challenges require multilateral responses, and we need allies and partners like Colombia to lead through the force of their example. You are already bringing significant expertise to bear in Central America and the Caribbean through our Action Plan on Regional Security, as well as your 2013 information sharing agreement with NATO, but we need Colombia's involvement in more places. 
Most importantly, Mr. President, our support for your efforts to negotiate peace in Colombia cannot be overstated. Just as we supported Colombia's leaders on the battlefield, we fully support you at the negotiating table. We acknowledge the significant challenges that lie ahead, and I applaud your efforts to take into account the rights of the more than six million victims of the conflict, your public pledge to prioritize human rights throughout this process, and your commitment to finding a balance between peace and justice that achieves a durable peace. The President and I look forward to our continued and candid discussions as you work toward a resolution of the conflict, and are hopeful for the historic day when all Colombians can live in a country free from fear and violence.

In closing, I offer you the full support of the U.S. government as you embark on yet another chapter of progress and development for the Colombian people. In that spirit, we look forward to seeing Colombia meet and exceed its own goals and continue to serve as an example of how a country can rise up from even the most difficult set of circumstances.

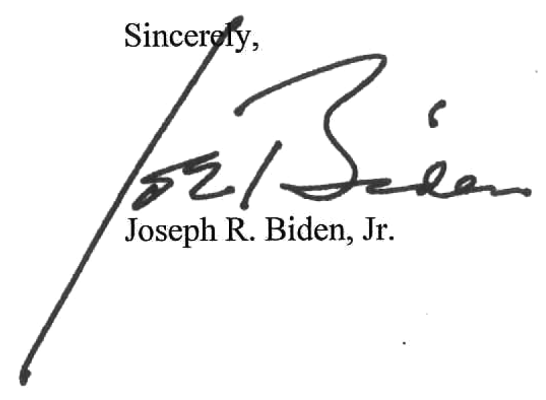

\section{REFERENCIAS}

1. European Medicines Agency. Assessment report: Remsima (infliximab). European Medicines Agency [internet] 2013 [acceso el 12 de febrero de 2017]. Disponible en: http:// www.ema.europa.eu/docs/en_GB/document_library/ EPAR_-_Public_assessment_report/human/002576/ WC $5001 \overline{5} 1486 . \mathrm{pdf}$.

2. Rickwood S, Iervolino A. Shaping the biosimilars opportunity: a global perspective on the evolving biosimilars landscape. IMS Health [internet] 2011 [acceso el 9 de junio de 2017]. Disponible en: https://weinberggroup.com/pdfs/ Shaping_the_biosimiliars_opportunity_A_global_perspective_on_the_evolving_biosimiliars_landscape.pdf.

3. Miller $\bar{K} L, \bar{L}$ Lanthier M. Regulatory watch: innovation in biologic new molecular entities: 1986-2014. Nat Rev Drug Discov. 2015;14(2):83. https://doi.org/10.1038/nrd4535

4. Gaviria A, Vaca González CP, Muñoz CG, et al. El debate de la regulación de medicamentos biotecnológicos: Colombia en el contexto mundial. Rev Panam Salud Publica. 2016;40(1):40-7.
5. Aitken M. Delivering on the potential of biosimilar medicines: The role of functioning competitive markets. IMS Health [internet] 2016 [acceso el 9 de junio de 2017]. Disponible en: http://www.imshealth.com/files/web/ IMSH\%20Institute/Healthcare\%20Briefs/DocDocume/ IMS_Institute_Biosimilar_Brief_March_2016.pdf.

6. von Schaper E. Celltrion's infliximab copy shows path to biosimilars in US. Nat Biotechnol. 2016;34(5):454-5. https:// doi.org/10.1038/nbt0516-454

7. Pavlovic M, Girardin E, Kapetanovic L, et al. Similar biological medicinal products containing recombinant human growth hormone: European regulation. Horm Res. 2008;69(1):14-21.

8. Danese S, Gomollón F. Governing Board and Operational Board of ECCO. ECCO position statement: the use of biosimilar medicines in the treatment of inflammatory bowel disease (IBD). J Crohns Colitis. 2013;7(7):586-9. https:// doi.org/10.1016/j.crohns.2013.03.011

9. GomollónF.Biosimilarsininflammatoryboweldisease: ready for prime time? Curr Opin Gastroenterol. 2015;31(4):2905. https://doi.org/10.1097/MOG.0000000000000184 
10. Danese S, Bonovas S, Peyrin-Biroulet L. Biosimilars in IBD: from theory to practice. Nat Rev Gastroenterol Hepatol. 2017;14(1):22-31. https://doi.org/10.1038/nrgastro.2016.155

11. Reinisch W, Louis E, Danese S. The scientific and regulatory rationale for indication extrapolation: a case study based on the infliximab biosimilar CT-P13. Expert Rev Gastroenterol Hepatol. 2015;9 Suppl 1:17-26. https://doi.org/10.1586/1 7474124.2015.1091306

12. Ha CY, Kornbluth A. A critical review of biosimilars in IBD: the confluence of biologic drug development, regulatory requirements, clinical outcomes, and big business. Inflamm Bowel Dis. 2016;22(10):2513-26. https://doi. org/10.1097/MIB.0000000000000886

13. Park W, Hrycaj P, Jeka S, et al. A randomised, double-blind, multicentre, parallel-group, prospective study comparing the pharmacokinetics, safety, and efficacy of CT-P13 and innovator infliximab in patients with ankylosing spondylitis: the PLANETAS study. Ann Rheum Dis. 2013;72(10):1605-12. https: / / doi.org/10.1136/annrheumdis-2013-eular.1548 https://doi.org/10.1136/annrheumdis-2012-203091

14. Yoo DH, Hrycaj P, Miranda P, et al. A randomised, doubleblind, parallel-group study to demonstrate equivalence in efficacy and safety of CT-P13 compared with innovator infliximab when coadministered with methotrexate in patients with active rheumatoid arthritis: the PLANETRA study. Ann Rheum Dis. 2013;72(10):1613-20. https://doi. org/10.1136/annrheumdis-2012-203090

15. Requisitos y el procedimiento para las evaluaciones farmacológica y farmacéutica de los medicamentos biológicos en el trámite del registro sanitario. Ministerio de Salud y Protección Social, decreto número 1782 del 18 de septiembre de 2014.

16. Argüelles-Arias F, Barreiro-de-Acosta M, Carballo F, et al. Joint position statement by "Sociedad Española de Patología Digestiva” (Spanish Society of Gastroenterology) and "Sociedad Española de Farmacología" (Spanish Society of Pharmacology) on biosimilar therapy for inflammatory bowel disease. Rev Esp Enferm Dig. 2013;105(1):37-43. https://doi.org/10.4321/S1130-01082013000100006

17. Targan SR, Hanauer SB, van Deventer SJ, et al. A shortterm study of chimeric monoclonal antibody cA2 to tumor necrosis factor alpha for Crohn's disease. Crohn's Disease cA2 Study Group. N Engl J Med. 1997;337(15):1029-35. https://doi.org/10.1056/NEJM199710093371502

18. Rutgeerts P, Vermeire S, Van Assche G. Biological therapies for inflammatory bowel diseases. Gastroenterology. 2009;136(4):1182-97. https:// doi.org/ 10.1053/ j.gastro. 2009.02.001

19. Pache I, Rogler G, Felley C. TNF-alpha blockers in inflammatory bowel diseases: practical consensus recommendations and a user's guide. Swiss Med Wkly. 2009;139(1920):278-87.

20. FordAC, Sandborn WJ, Khan KJ, et al.Efficacy of biological therapies in inflammatory bowel disease: systematic review and metaanalysis. Am J Gastroenterol. 2011;106(4):644-59. https://doi. org/10.1038/ajg.2011.73 https://doi.org/10.1038/ajg.2011.71 https://doi.org/10.1038/ajg.2011.70

21. Chingcuanco F, Segal JB, Kim SC, et al. Bioequivalence of biosimilar tumor necrosis factor- $\alpha$ inhibitors compared with their reference biologics: a systematic review. Ann Intern Med. 2016;165(8):565-74. https://doi.org/10.7326/M160428

22. Schellekens H. Biosimilar therapeutics-what do we need to consider? NDT Plus. 2009;2(Suppl_1):i27-36.

23. Roger SD. Biosimilars: how similar or dissimilar are they? Nephrology (Carlton). 2006;11(4):341-6. https://doi. org/10.1111/j.1440-1797.2006.00594.x

24. Kozuch PL, Hanauer SB. General principles and pharmacology of biologics in inflammatory bowel disease. Gastroenterol Clin North Am. 2006;35(4):757-73.

25. Park W, Lee SJ, Yun J, et al. Comparison of the pharmacokinetics and safety of three formulations of infliximab (CTP13, EU-approved reference infliximab and the US-licensed reference infliximab) in healthy subjects: a randomized, double-blind, three-arm, parallel-group, single-dose, Phase I study. Expert Rev Clin Immunol. 2015; 11 Suppl 1:S25-31. https://doi.org/10.1586/1744666X.2015.1090311

26. Devlin SM, Bressler B, Bernstein CN, et al. Overview of subsequent entry biologics for the management of inflammatory bowel disease and Canadian Association of Gastroenterology position statement on subsequent entry biologics. Can J Gastroenterol. 2013;27(10):567-71. https://doi.org/10.1155/2013/327120

27. Health Canada. Regulatory decision summary inflectra supplemental new drug submission 2016/06/10. 2016 [acceso el 12 febrero de 2017]. Disponible en: https:// www.canada.ca/en/health-canada/services/publications/ drugs-health-products/health-canada-new-drug-authorizations-2016-highlights.html

28. Vande Casteele N, Sandborn WJ. IBD: Indication extrapolation for anti-TNF biosimilars. Nat Rev Gastroenterol Hepatol. 2015;12(7):373-4.

29. Feagan BG, Choquette D, Ghosh S, Gladman DD, Ho V, Meibohm B, Zou G, Xu Z, Shankar G, Sealey DC, Russell AS. The challenge of indication extrapolation for infliximab biosimilars. Biologicals. 2014 Jul;42(4):177-83. https:// doi.org/10.1016/j.biologicals.2014.05.005

30. Levin AD, Wildenberg ME, van den Brink GR. Mechanism of action of anti-TNF therapy in inflammatory bowel disease. J Crohns Colitis. 2016;10(8):989-97. https://doi. org/10.1093/ecco-jcc/jjw053

31. Food and Drug Administration. Nonpropietary naming of biological products. FDA [internet] 2017 [acceso el 12 febrero de 2017]. Disponible en: https://www.fda.gov/ downloads/drugs/guidances/ucm459987.pdf.

32. Food and Drug Administration. Designation of official names and proper names for certain biological products: proposed rule. FDA [internet] 2015 [acceso el 12 febrero de 2017]. Disponible en: https://www.gpo.gov/fdsys/pkg/ FR-2015-08-28/pdf/2015-21382.pdf. 
33. European Commission. Consensus Information Paper 2013. What you need to know about Biosimilar Medicinal Products. European Commission [internet] 2013 [acceso el 12 febrero de 2017]. Disponible en: http://www.medicinesforeurope.com/wp-content/uploads/2016/03/biosimilars_report_en.pdf.

34. Vermeer NS, Spierings I, Mantel-Teeuwisse AK, et al. Traceability of biologicals: present challenges in pharmacovigilance. Expert Opin Drug Saf. 2015;14(1):63-72. https://doi.org/10.1517/14740338.2015.972362

35. Dörner T, Strand V, Castañeda-Hernández G, et al. The role of biosimilars in the treatment of rheumatic diseases. Ann Rheum Dis. 2013;72(3):322-8. https://doi.org/10.1136/ annrheumdis-2012-202715

36. Casadevall N, Nataf J, Viron B, et al. Pure red-cell aplasia and antierythropoietin antibodies in patients treated with recombinant erythropoietin. N Engl J Med. 2002;346(7):469-75. https://doi.org/10.1056/NEJMoa011931

37. Ben-Horin S, Yavzori M, Benhar I, et al. Crossimmunogenicity: antibodies to infliximab in Remicadetreated patients with IBD similarly recognise the biosimilar Remsima. Gut. 2016;65(7):1132-8.

38. Márquez Velásquez JR. Inmunogenicidad cruzada: anticuerpos para infliximab en pacientes con enfermedad inflamatoria intestinal tratados con Remicade reconocen similarmente al biosimilar Remsima. Enfermedad Inflamatoria Intestinal al Día. 2016;15:19-21. https://doi.org/10.1016/j. eii.2015.12.002

39. Kang YS, Moon HH, Lee SE, etal. Clinical experience of the use of CT-P13, a biosimilar to infliximab in patients with inflammatory bowel disease: A Case Series. Dig Dis Sci. 2015;60(4):951-6. https://doi.org/10.1007/s10620-014-3392-z

40. Jung YS, Park DI, Kim YH, et al. Efficacy and safety of CT-P13, a biosimilar of infliximab, in patients with inflammatory bowel disease: A retrospective multicenter study. J Gastroenterol Hepatol. 2015;30(12):1705-12. https://doi. org/10.1111/jgh.12997

41. Hlavaty T, Krajcovicova A, Sturdik I, et al. Biosimilar infliximab CT-P13 treatment in patients with inflammatory bowel disease - a one-year, single centre retrospective study. Journal of Gastroenterology and Hepatology. 2016;70(1):27-36. https://doi.org/10.14735/amgh201627

42. Jahnsen J, Detlie TE, Vatn S, et al. Biosimilar infliximab (CT-P13) in the treatment of inflammatory bowel disease: A Norwegian observational study. Expert Rev Gastroenterol Hepatol. 2015;9 Suppl 1:45-52. https://doi.org/10.1586/1 7474124.2015.1091308

43. Gecse KB, Lovász BD, Farkas K, et al. Efficacy and safety of the biosimilar infliximab ct-p13 treatment in inflammatory bowel diseases: a prospective, multicentre, nationwide cohort. J Crohns Colitis. 2016;10(2):133-40.

44. Bálint A, Rutka M, Végh Z, et al. Frequency and characteristics of infusion reactions during biosimilar infliximab treatment in inflammatory bowel diseases: results from Central European nationwide cohort. Expert Opin Drug Saf. $2017 ; 16(8): 885-890$.
45. Jørgensen Ket al. (2016) 'LB15 - Biosimilar infliximab (CT$\mathrm{P} 13$ ) is not inferior to originator infliximab: results from the 52-week randomized NOR-SWITCH trial.' Abstract presented at the United European Gastroenterology (UEG) Week meeting 2016;15-19.

46. Faccin F, Tebbey P, Alexander E, Wang X, Cui L, Albuquerque $\mathrm{T}$. The design of clinical trials to support the switching and alternation of biosimilars. Expert Opin Biol Ther. 2016;16(12):1445-53.

47. Farkas K, Rutka M, Golovics PA, et al. Efficacy of Infliximab Biosimilar CT-P13 Induction Therapy on Mucosal Healing in Ulcerative Colitis. J Crohns Colitis. 2016;10(11):12731278.

48. Rencz F, Brodszky V, Baji P, et al. Cost-utility of biosimilar infliximab (Inflectra (C) for the treatment of luminal crohn's disease in nine European countries. United European Gastroenterology Journal. 2016;2 (Supplement 1).

49. Severs M, Oldenburg B, van Bodegraven AA, et al. The economic impact of the introduction of biosimilars in inflammatory bowel disease. J Crohns Colitis. 2017;11(3):289-96.

50. Annese V, Vecchi M. Italian Group for the Study of IBD (IG-IBD). Use of biosimilars in inflammatory bowel disease: statements of the italian group for inflammatory bowel disease. Dig Liver Dis. 2014;46(11):963-8. https:// doi.org/10.1016/j.dld.2014.07.019

51. Danese S, Fiorino G, Raine T, et al. ECCO position statement on the use of biosimilars for inflammatory bowel disease-an update. J Crohns Colitis. 2017;11(1):26-34. https://doi.org/10.1093/ecco-jcc/jjw198

52. Crohn's and Colitis Foundation of America Position Statement on Biosimilars. Febrero 9 de 2016 [acceso el 11 de diciembre de 2017]. Disponible en: http://www. crohnscolitisfoundation.org/assets/pdfs/advocacy/ccfacomments-to-fda-re.pdf.

53. British Society of Gastroenterology. BSG Guidance on the use of biosimilar infliximab CT-P13 in inflammatory bowel disease. BSG [internet] 2016 [acceso el 22 de enero de 2017]. Disponible en: http://www.bsg.org.uk/images/stories/ docs/clinical/guidance/bsg_infliximab_guidagui_16.pdf.

54. World Health Organization. Biological qualifier: an INN proposal. WHO [internet] 2014 [acceso el 12 febrero de 2017]. Disponible en: http://www.who.int/medicines/services/inn/bq innproposal201407.pdf.

55. European Commission, Health and Consumers Directorate General [acceso el 11 de diciembre de 2017]. Disponible en: www.ema.europa.eu/docs/en_GB/document_library/ Report/2017/12/WC500239794.pdf

56. Annese V, Avendaño-Solá C, Breedveld F, et al. Roundtable on biosimilars with European regulators and medical societies, Brussels, Belgium, 12 January 2016. GaBI Journal. 2016;5(2):74-83. https://doi.org/10.5639/ gabij.2016.0502.019 